\title{
Biotechnological uses of RNAi in plants: risk assessment considerations
}

\author{
Josep M. Casacuberta ${ }^{1}$, Yann Devos ${ }^{2}$, Patrick du Jardin ${ }^{3}$, Matthew Ramon ${ }^{2}$, \\ Hervé Vaucheret ${ }^{4,5}$, and Fabien Nogué ${ }^{4,5}$
}

\author{
${ }^{1}$ Center for Research in Agricultural Genomics, Consejo Superior de Investigaciones Científicas-Institut de Recerca i Tecnologia \\ Agroalimentàries-Universitat Autònoma de Barcelona-Universitat de Barcelona, Campus Universitat Autònoma de Barcelona, \\ Bellaterra - Cerdanyola del Vallès, 08193, Barcelona, Spain \\ ${ }^{2}$ European Food Safety Authority, Via Carlo Magno 1/A, 43126 Parma, Italy \\ ${ }^{3}$ Gembloux Agro-Bio Tech, Plant Biology Unit, University of Liège, Gembloux, Belgium \\ ${ }^{4}$ INRA, Institut Jean-Pierre Bourgin, Saclay Plant Sciences, Versailles, France \\ ${ }^{5}$ AgroParisTech, Institut Jean-Pierre Bourgin, Saclay Plant Sciences, Versailles, France
}

\begin{abstract}
RNAi offers opportunities to generate new traits in genetically modified (GM) plants. Instead of expressing novel proteins, RNAi-based GM plants reduce target gene expression. Silencing of off-target genes may trigger unintended effects, and identifying these genes would facilitate risk assessment. However, using bioinformatics alone is not reliable, due to the lack of genomic data and insufficient knowledge of mechanisms governing mRNA-small (s)RNA interactions.
\end{abstract}

\section{Background}

Technological advances in genetics and genomics led to the development of new types GM plant in which target genes are silenced through RNAi. RNAi is a conserved mechanism that relies on the production of sRNA (20-30 nucleotides in size), which promotes degradation or translation repression of homologous mRNA. In the common eukaryotic ancestor, RNAi was probably meant to target molecular parasites, such as viruses or transposons [1], but subsequently evolved to act on various aspects of gene regulation or genome structure [2]. RNAi can occur naturally, as a result of natural modifications of gene copy number or genetic arrangements. Plant breeders have already selected for natural RNAi-mediated traits, such as seed coat colour or low glutelin level, in soybean and rice, respectively [3], through conventional breeding without the aid of any transgenic manipulation.

However, transgenic RNAi has emerged as a powerful and innovative genetic tool for biological research. It has been utilised in fundamental research for the assessment of gene functions, and in various fields of applied research, such as human and/or veterinary medicine, and agriculture. Given its potential to control gene expression, RNAi offers new opportunities for plant breeding (Box 1), and is being used to alter agronomic and compositional characteristics in GM plants (e.g., nutritional enrichment,

Corresponding author: Nogué, F. (fabien.nogue@versailles.inra.fr).

Keywords: food/feed safety; gene silencing; genetically modified organism (GMO); nontarget organism (NTO); off-target gene; plant breeding.

0167-7799/

(c) 2014 Elsevier Ltd. All rights reserved. http://dx.doi.org/10.1016/j.tibtech.2014.12.003 antinutrient reduction, and disease or pest resistance) [4]. RNAi-based GM plants are approaching commercialisation and, similar to GM plants in most jurisdictions, will likely be subject to a risk analysis before regulatory approval. Several international risk assessment bodies involved in the pre-market risk assessment of GM plants are currently considering whether existing approaches are appropriate or need to be refined for RNAi-based GM plants (Table 1). Here, we discuss possible risks to human and animal health and the environment associated with the silencing of off-target genes, and possible strategies to assess these risks.

\section{Risk assessment considerations for RNAi-based GM plants}

Risks associated with the intended changes in current GM plants are usually related to the newly expressed proteins (e.g., possible toxicity and allergenicity). Given that most RNAi strategies aim to reduce the amount of endogenous proteins, such risks are not necessarily relevant for RNAibased GM plants. However, the decreased expression of a target gene may have safety implications in particular cases (e.g., if a substrate of a silenced enzyme accumulates to toxic levels).

Risks more specifically associated with RNAi strategies exist. Given that RNAi activity is based on the pairing between sRNA and mRNA, not only the target gene, but also off-target genes that produce mRNA with sufficient nucleotide sequence complementarity to the sRNA could be silenced [5]. Owing to the conserved nature of RNAi [6], target and off-target genes could be silenced in both the RNAi-based GM plant and organisms consuming and/or infecting that plant. These organisms include target pests, in the case of host-delivered RNAi, and nontarget organisms (NTOs). Silencing of target and off-target genes in the GM plant and NTOs could lead to adverse effects, which require assessment. However, the likelihood of silencing a given gene will depend on the ability of the sRNA to be a substrate for the RNAi machinery of the recipient organism. Indeed, RNAi machinery varies among species [7] and there is convincing evidence for only a few organisms (e.g., nematodes and insects) that the plant-produced sRNA uses host RNAi machinery [8]. It should be noted that 
Box 1. Strengths and limitations of RNAi as a tool for plant breeding

\section{Strengths}

- The efficacy of silencing of a target gene via RNAi varies from one GM plant to another [13], which allows for the identification of plants showing a range of decreased target gene expression. This is particularly interesting when complete silencing of the target gene is not viable or induces an undesirable phenotype. This may be particularly interesting for the reduction of antinutrient levels in GM plants.

- The silencing of a target gene via RNAi can be spatially and/or temporally controlled by suitable promoters, in contrast to a conventional gene knockout. It is possible to downregulate a gene function in particular tissues and/or organs, while preserving it in other tissues and/or organs or developmental stages.

- Many important crop species have genomes originating from ancestral or recent polyploidisation events and, therefore, contain multiple alleles of a gene; RNAi enables their simultaneous silencing [14].

- The RNAi process is, in part, ubiquitous among eukaryotes, and double-stranded RNA or small interfering RNA (siRNA) produced by a plant can, in some cases, silence a target gene in an organisms feeding on or infecting that plant. This offers new opportunities for the control of pests and plant diseases [15].

\section{Limitations}

- For reasons that are still not well understood, some mRNAs appear difficult to silence through RNAi [13].
- Given that rules governing the pairing of sRNAs with target mRNAs are not fully known, off-target gene effects are possible.

- Besides triggering the degradation or translation repression of homologous mRNA, RNAi also induces the methylation of homologous DNA, including the transcribed region of RNAi transgene itself. DNA methylation sometimes spreads into adjacent sequences such as the transgene promoter, which could eventually reduce RNAi efficiency over generations.

- Given that RNAi relies on the sequence-specific pairing of sRNA to mRNA, mutations arising in the target mRNA in the middle of its complementary region to the sRNA guide could prevent targeting this $\mathrm{mRNA}$, sometimes without changing the translated amino acid sequence (silent mutations). Such mutations act in a dominant manner, which increases the likelihood of occurrence in nature. This is of particular importance for RNAi-mediated pest or disease control if RNAi relies on the production of a single artificial miRNA and not on a population of siRNA. For example, mutations in the target sequence enable viruses to circumvent silencing mediated by an artificial miRNA produced in GM plants.

- Mutations arising in an endogenous mRNA, close, but not perfectly homologous to the target mRNA, could create a complementary region. Having a novel target in GM plants would dilute the effect of transgene-derived sRNA(s) on the expected target. Such mutations would also act in a dominant manner. not all off-target interactions will result in a significant reduction of in vivo gene expression. Even in the event that the expression level of a gene is reduced, this may not necessarily result in detectable phenotypic changes. Only a fraction of such unexpected phenotypic changes may translate into adverse effects, depending on the function of the silenced off-target gene.

Given that silencing off-target genes within the GM plant or in organisms infecting or feeding on the plant may, in some cases, constitute a new risk for humans, other animals, or the environment, such risks should be properly assessed and managed. Genome-wide bioinformatic analyses of sequence complementarity between the expressed sRNA(s) and all known mRNAs would help to identify potential off-target genes in the GM plant itself and in NTOs. However, there are several limitations to such analyses. Despite the large number of sequenced genomes, the genomes of many crop plants remain unknown. In addition, for those genomes that have been sequenced, information is still limited, because variable fractions, usually comprising centromeric or highly repetitive parts of the genome, remain unknown.

For most sequenced species, only a reference genome is sequenced and assembled to such an extent that it could be considered relatively complete. Given that the sRNAmRNA interaction relies on a limited sequence length, any sequence variability between varieties may change the spectrum of potential target and off-target genes. Owing to the lack of genome sequence information for all the varieties in which the RNAi-based transformation event may be introduced, it will not be possible to perform a complete search of all potential off-target genes. In addition, because various species belonging to different taxa can be exposed to an RNAi-based GM plant [9], the limited availability of genome data for non-model species further reduces the usefulness of a bioinformatics-based approach for the risk assessment of NTOs. Owing to these limitations, bioinformatic analyses are likely to be of limited value to predict off-target gene effects in the GM plant or in NTOs exposed to that plant in most cases [10]. However, bioinformatics could inform the species selection process by identifying groups of NTOs that are potentially sensitive.

A complementary approach to bioinformatics analyses could be to use new molecular techniques, such as parallel analysis of RNA ends (PARE) [11]. PARE relies on the ability of cleaved RNAs, such as those resulting from sRNA-guided cleavage of a mRNA by the RNAi machinery, to ligate to RNA adaptors by virtue of the absence of a cap at their $5^{\prime}$ end (capped mRNAs cannot be ligated). Sequencing libraries of cleaved mRNAs from both the RNAi-based GM plant and an adequate comparator would allow the determination of the intended and unintended targets of the sRNA(s) produced by the RNAi construct. However, because the population of mRNAs depends on the cell types, organs, developmental stages, or environmental conditions, a complete evaluation of possible off-target genes remains challenging. Moreover, this inventory of unintended targets of the sRNA(s) produced by the RNAi construct would be restricted to the GM plant itself and not likely feasible in NTOs.

\section{Concluding remarks and future perspectives}

RNAi is an emerging technology that offers new opportunities to plant breeders. The risk assessment of RNAibased GM plants presents some peculiarities compared with that of currently commercialised GM plants. Allergenicity assessment of newly expressed proteins, for example, will not be meaningful for RNAi-based GM plants. By contrast, the evaluation of the potential risk associated with the silencing of an off-target gene is specific to this 
Table 1. Risk assessment considerations on RNAi-based GM plants and derived food and/or feed products from international bodies

Authority and/or institute

Center for Environmental Risk Assessment (CERA)

European Food Safety Authority (EFSA)

Food Standards Australia New Zealand (FSANZ)

US Environmental Protection Agency (US EPA)
Web address

http://cera-gmc.org/docs/cera_publications/pub_08_2011.pdf

http://www.efsa.europa.eu/fr/events/event/140604.htm

http://www.foodstandards.govt.nz/consumer/gmfood/Documents/

Heinemann\%20Response\%20210513.pdf

http://www.epa.gov/scipoly/sap/meetings/2014/january/012814minutes.pdf technology. The effects of off-target gene silencing that significantly impact agronomic performance and crop quality can be detected, and counter-selected, by the breeder during the breeding process, as is the case for the development of any new trait in GM and conventional plants, but other unintended effects could persist in the final RNAibased plant. The identification of such potential off-target effects could be achieved by the identification of mRNAs that exhibit limited, although significant, complementary to the sRNA molecules produced by the RNAi construct. Although new bioinformatics [12] and molecular techniques [11] may help to evaluate the spectrum of off-target sequences, these analysis are currently of limited value for the risk assessment for three reasons: (i) suitable genome data are available only for a limited number of species; (ii) rules governing efficient $\mathrm{mRNA}$ and/or sRNA recognition by the RNAi machinery are incompletely understood; and (iii) the capacity of plant sRNA to trigger silencing in nonplant organisms is not always clear and has been estimated for only a few species. Progress in basic research on RNAi mechanisms, production of suitable genome data for relevant species, and design of efficient algorithms to make more reliable predictions will help to fill these technological gaps. This new information and the associated tools will not only facilitate the risk assessment of RNAi-based GM plants, but may also have a more general impact on the evaluation of potential risk associated with the production of sRNA in GM or non-GM plants. Despite these technical limitations and possible advancements, the risk assessment strategies followed for current GM plants, based on the comparative analysis of the molecular, compositional, and agronomic/phenotypic characteristics of the GM plant and its conventional counterpart, remain applicable and adequate for the evaluation of RNAi-based GM plants.

\section{Acknowledgements}

The authors would like to thank Elisabeth Waigmann for critical reading of the manuscript. F.N. was supported by the Agence National de la Recherche (program ANR-11-BTBR-0001-GENIUS).

\section{Disclaimer statement}

Y.D. and M.R. are employed by EFSA. However, the views or positions expressed in this publication do not necessarily represent in legal terms the official position of EFSA. EFSA assumes no responsibility or liability for any errors or inaccuracies that may appear.

\section{References}

1 Obbard, D.J. et al. (2009) The evolution of RNAi as a defence against viruses and transposable elements. Philos. Trans. R. Soc. Lond. B: Biol. Sci. 364, 99-115

2 Castel, S.E. and Martienssen, R.A. (2013) RNA interference in the nucleus: roles for small RNAs in transcription, epigenetics and beyond. Nat. Rev. Genet. 14, 100-112

3 Erdmann, V.A. and Barciszewski, J. (2011) Non-Coding RNAs in Plants, Springer

4 Katoch, R. and Thakur, N. (2013) Advances in RNA interference technology and its impact on nutritional improvement, disease and insect control in plants. Appl. Biochem. Biotechnol. 169, 1579-1605

5 Senthil-Kumar, M. and Mysore, K.S. (2011) Caveat of RNAi in plants: the off-target effect. Methods Mol. Biol. 744, 13-25

6 Knip, M. et al. (2014) Trans-kingdom cross-talk: small RNAs on the move. PLoS Genet. 10, e1004602

7 Saumet, A. and Lecellier, C.H. (2006) Anti-viral RNA silencing: do we look like plants? Retrovirology 3,3

8 Dua, C-G. et al. (2012) Application of RNA silencing to plant disease resistance. Silence 3, 5

9 Romeis, J. et al. (2014) Potential use of an arthropod database to support the non-target risk assessment and monitoring of transgenic plants. Transgenic Res. 23, 995-1013

10 Lundgren, J.G. and Duan, J.J. (2013) RNAi-based insecticidal crops. Bioscience 63, 657-665

11 German, M.A. et al. (2008) Global identification of microRNA-target RNA pairs by parallel analysis of RNA ends. Nat. Biotechnol. 26, 941-946

12 Zhong, R. et al. (2014) Computational detection and suppression of sequence-specific off-target phenotypes from whole genome RNAi screens. Nucleic Acids Res. 42, 8214-8222

13 Kerschen, A. et al. (2004) Effectiveness of RNA interference in transgenic plants. FEBS Lett. 566, 223-228

14 Rathore, K.S. et al. (2012) Ultra-low gossypol cottonseed: generational stability of the seed-specific, RNAi-mediated phenotype and resumption of terpenoid profile following seed germination. Plant Biotechnol. J. 10, 174-183

15 Runo, S. (2011) Engineering host-derived resistance against plant parasites through RNA interference: challenges and opportunities. Bioeng. Bugs 2, 208-213 